\title{
Continuous-infusion Ifosfamide and Doxorubicin Combination as Second-Line Chemotherapy for Recurrent or Refractory Osteosarcoma Patients in China: a Retrospective Study
}

\author{
Yu-Jing Huang, Ai-Na He, Yuan-Jue Sun, Zan Shen, Da-Liu Min, Yang Yao*
}

\begin{abstract}
Objective: The aim of this retrospective study was to evaluate the feasibility and efficacy of response to continuous-infusion ifosfamide and doxorubicin combination as second-line chemotherapy for patients with recurrent or refractory osteosarcoma. Materials and Methods: Eighteen recurrent or refractory osteosarcoma patients who were treated with continuous-infusion ifosfamide and doxorubicin combination between May 1999 and April 2011 were included in the analysis. Ifosfamide at $12 \mathrm{~g} / \mathrm{m}^{2}$ was administered by intravenous continuous infusion over 3 days, and doxorubicin $60 \mathrm{mg} / \mathrm{m}^{2}$ was administered as an intravenous bolus injection on day 1 . The combination therapy was repeated every 3 weeks. Treatment was continued until evidence of disease progression or unacceptable toxicity. Results: The patients (ages 7-53 years) received a total of 42 cycles of chemotherapy (median: 2 courses; range: $2-5$ courses). The overall response rate was $0 \%$ and the disease control rate was $22.3 \%$, with four patients having stable disease. The median time to progression and overall survival time were 2 months (range: 2-5 months) and 9 months (range: 3-29 months), respectively. Major severe toxicities were leucopenia $7(38.9 \%)$, nausea and vomiting $3(16.7 \%)$ and alopecia $9(50 \%)$. There were no treatment-related deaths. Conclusions: In our experience, continuous-infusion ifosfamide and doxorubicin combination therapy at this dosage and schedule was found to be well tolerated and moderate effective, which could be considered as salvage therapy for patients with recurrent or refractory osteosarcoma. Further assessment is necessary to confirm the safety and efficacy of this treatment.
\end{abstract}

Keywords: Continuous-infusion - ifosfamide - doxorubicin - salvage chemotherapy - osteosarcoma

Asian Pac J Cancer Prev, 16 (6), 2391-2395

\section{Introduction}

Osteosarcoma is the most common malignant primary bone tumor predominantly affecting young people, comprising about $20 \%$ of primary bone sarcoma (Ta et al., 2009). The introduction of multi-disciplinary treatment (surgical resection in conjunction with perioperative multi-agent chemotherapy) improved the survival of osteosarcoma patients dramatically (Sluga et al., 1999; Bacci et al., 2002; Hawkins et al., 2003; Wilkins et al., 2003; Kudawara et al., 2013). However, the prognosis of patients who present with unresectable or relapsed disease remains poor (Bielack et al., 2002; Ferrari et al., 2003). Studies showed that second line chemotherapy might improve the outcome of these patients (Saeter et al., 1995; Ferrari et al., 2003). However, the optimal treatment strategy for these patients is still not defined.

The key drugs in the standard chemotherapy for osteosarcoma include high-dose methotrexate, cisplatin and doxorubicin (Meyers et al., 2005). Ifosfamide is also reported to be an active agent for osteosarcoma by the schedule of a bolus dose in 30-60 min over 2-5 days (Harris et al., 1995; Kobys et al., 2013). Recently, several retrospective clinical trials had been conducted to assess the efficacy and toxicity of continuous-infusion ifosfamide-based combination therapy for sarcomas, but the results were controversial. And what's more, pathologic histologies of the included patients, the dosages of ifosfamide and chemotherapy schedules in the studies were diverse (Palumbo et al., 1997; Singer et al., 1998; Cartei et al., 2003; De Pas et al., 2011).

Therefore, the role of continuous-infusion ifosfamidebased combination therapy in refractory or recurrent high-grade osteosarcoma is still not well defined. As a result, we undertake this retrospective study to assess the efficacy and toxicity of continuous-infusion ifosfamide and doxorubicin combination therapy for recurrent or refractory high-grade osteosarcoma. As far as we know, this is the largest retrospective study so far to investigate continuous-infusion ifosfamide and doxorubicin combination therapy for recurrent or refractory osteosarcoma patients.

Department of Oncology, Affiliated Sixth People's Hospital, Shanghai Jiaotong University, Shanghai, China *For correspondence: yangyao12@yahoo.com 


\section{Materials and Methods}

\section{Patients and treatment}

Eighteen patients with relapsed and refractory osteosarcoma who received continuous-infusion ifosfamide and doxorubicin combination therapy as second-line chemotherapy at our institution between May 1999 and April 2011 were selected for this retrospective case series study, according to the following criteria:i) histological confirmation of diagnosis; $i$ ) prior treatment (completed $>3$ weeks before trial entry) consisted of standard high-grade osteosarcoma chemotherapy agents including doxorubicin, cisplatin, ifosfamide, and high-dose methotrexate; iii) disease progression with radiological evidence; $i v$ ) Karnofsky performance status $\geq 70$ with a life expectancy $>3$ months; $v$ ) adequate renal, hepatic, and hemopoietic function. The patients' clinical characteristics such as age, gender, pathologic subtypes, and performance status were collected for statistical analysis.

Ethical approval for the study was provided by the independent ethics committee, Sixth people's Hospital, Shanghai JiaoTong University. Informed and written consent was obtained from all patients or their advisers according to ethics committee guidelines.

The ifosfamide-doxorubicin regimen was administered as follows: $i$ ) ifosfamide $12 \mathrm{~g} / \mathrm{m}^{2}$ i.v. as continuous infusion over 3 days; $i$ i) mesna $16 \mathrm{~g} / \mathrm{m}^{2}$ i.v. as continuous infusion over 4 days; iii) doxorubicin $60 \mathrm{mg} \mathrm{g} / \mathrm{m}^{2}$ i.v. bolus on day 1 . Every 3 -week treatment schedule was designated as one cycle, and a cycle of chemotherapy was given every 3 weeks. Prophylactic 5-hydroxytryptamine (5-

\section{Table 1. Patient Characteristics}

\begin{tabular}{|c|c|c|}
\hline Features & $\mathrm{n}$ & percent \\
\hline \multicolumn{3}{|l|}{ Gender } \\
\hline Male & 14 & 77.8 \\
\hline Female & 4 & 22.2 \\
\hline \multicolumn{3}{|l|}{ Age at study entry (years) } \\
\hline Median (range) & \multicolumn{2}{|c|}{$16.5(7-53)$} \\
\hline$<18$ & 10 & 55.6 \\
\hline$\geq 18$ & 8 & 44.4 \\
\hline \multicolumn{3}{|l|}{ KPS } \\
\hline 90 & 6 & 33.3 \\
\hline 80 & 9 & 50 \\
\hline 70 & 3 & 16.7 \\
\hline \multicolumn{3}{|l|}{ Location of primary tumor } \\
\hline Upper limbs & 5 & 27.8 \\
\hline Lower limbs & 11 & 61.1 \\
\hline Non-extremities & 2 & 11.1 \\
\hline \multicolumn{3}{|l|}{ Histotype } \\
\hline Conventional & 15 & 83.3 \\
\hline Other & 3 & 16.7 \\
\hline \multicolumn{3}{|l|}{ Metastatic at diagnosis } \\
\hline Yes & 5 & 27.8 \\
\hline No & 13 & 72.2 \\
\hline \multicolumn{3}{|l|}{ Adjuvant chemotherapy } \\
\hline Yes & 12 & 66.7 \\
\hline No & 6 & 33.3 \\
\hline \multicolumn{3}{|l|}{ Patterns of relapse } \\
\hline Lung metastasis & 11 & 61.1 \\
\hline Lung metastasis + local recurrence & 3 & 16.7 \\
\hline Lung metastasis + bone metastasis & 1 & 22.2 \\
\hline
\end{tabular}

HT3) receptor antagonists and other antiemetic treatments were given to all patients. Granulocyte colony-stimulating factor was used when patients with febrile neutropenia or Grade 4 neutropenia were judged as requiring its administration by physician-in-charge.

\section{Patient assessment}

Tumor response was usually evaluated every two chemotherapy cycles by computed tomography (CT)/ magnetic resonance imaging (MRI) scan according to the Response Evaluation Criteria in Solid Tumors (RECST) (Therasse et al., 2000). Treatment responses were classified as: $i$ ) complete response $(\mathrm{CR}), i i)$ partial response (PR), iii) progression disease (PD), and $i v$ ) stable disease (SD). Only patients with $\mathrm{SD}, \mathrm{PR}$, and CR continued chemotherapy.

The maximum toxicity was recorded for each cycle of chemotherapy according to the National Cancer InstituteCommon Toxicity Criteria (NCI-CTC, version 3.0). All toxicities were classified into four levels; the following toxicities were recorded: white blood cell count, platelet count, hemoglobin, gastrointestinal toxicities (nausea and vomiting), fatigue, alopecia, impaired liver function, impaired renal function, cardiotoxicity, and hematuresis.

\section{Statistical analysis}

A retrospective analysis in terms of response rate, progression-free survival (PFS) and overall survival (OS) was performed. The Kaplan-Meier method was used to estimate the PFS and the OS. OS was defined as the time from the start of treatment to death from any cause. Patients alive at the time of analysis were censored at the date of last follow-up. PFS was measured from the start of treatment to progression of the disease or death from any cause. Patients who were alive and progression free at the last follow-up were censored. Duration of response was defined as time from the date of the first objective response until the time of patient progression or death. Data were presented as percentage or median plus range unless otherwise specified. All analyses were performed with SPSS soft-ware version 13.0 (SPSS, Inc., Chicago, IL, USA).

\section{Results}

\section{Patient Characteristics}

Between May 1999 and April 2011, 18 patients with relapsed and refractory osteosarcoma were treated with continuous-infusion ifosfamide and doxorubicin combination therapy in our institution. Table 1 lists the features of the analyzed patients. The patients' median age was 16.5 years (range 7-53 years), and $77.8 \%$ (14/18) were male. Most patients (83.3.0\%) had a KPS of $\geq 80$, and $27.8 \%$ had metastatic disease at diagnosis. After primary therapy, eleven patients had lung metastasis alone, three patients had local recurrence with lung metastasis and four patients had bone metastasis with lung metastasis. $66.7 \%$ received adjuvant chemotherapy.

Efficacy and safety

All patients received at least two cycles of continuous- 
Table 2. Details of Response to Continuous-iccccnfusion Ifosfamide and Doxorubicin Chemotherapy

\begin{tabular}{|c|c|c|c|c|c|c|c|}
\hline $\begin{array}{l}\text { Patient } \\
\text { number }\end{array}$ & $\begin{array}{l}\text { Age } \\
\text { (year) }\end{array}$ & Sex & $\begin{array}{l}\text { Site of } \\
\text { primary }\end{array}$ & $\begin{array}{l}\text { Metastases } \\
\text { at diagnosis }\end{array}$ & $\begin{array}{l}\text { Site of } \\
\text { relapse }\end{array}$ & $\begin{array}{c}\text { Number of } \\
\text { cycles }\end{array}$ & $\begin{array}{l}\text { Best response to } \\
\text { continuous-infusion ifosfamide } \\
\text { and doxorubicin combination therapy }\end{array}$ \\
\hline 1 & 7 & Male & Tibia & No & $\mathrm{LM}+\mathrm{BM}$ & 2 & PD \\
\hline 2 & 12 & Male & Tibia & No & $\mathrm{LM}+\mathrm{BM}$ & 2 & PD \\
\hline 3 & 25 & Male & Humerus & No & LM & 3 & SD \\
\hline 4 & 16 & Male & Tibia & No & LM & 2 & $\mathrm{PD}$ \\
\hline 5 & 17 & Male & Femur & No & LM & 5 & $\mathrm{SD}$ \\
\hline 6 & 43 & Male & Femur & Lung & $\mathrm{LM}+\mathrm{LR}$ & 3 & $\mathrm{SD}$ \\
\hline 7 & 16 & Male & Tibia & Lung & LM & 2 & PD \\
\hline 8 & 36 & Male & Ribs & No & LM & 2 & $\mathrm{PD}$ \\
\hline 9 & 41 & Male & Maxilla & Lung & $\mathrm{LM}+\mathrm{LR}$ & 2 & PD \\
\hline 10 & 12 & Female & Femur & Lung & LM & 3 & $\mathrm{SD}$ \\
\hline 11 & 16 & Female & Humerus & No & LM & 2 & $\mathrm{PD}$ \\
\hline 12 & 53 & Male & Humerus & No & LM & 2 & PD \\
\hline 13 & 15 & Female & Tibia & No & LM & 2 & PD \\
\hline 14 & 15 & Male & Femur & Lung & LM & 2 & PD \\
\hline 15 & 20 & Male & Humerus & No & $\mathrm{LM}+\mathrm{LR}$ & 2 & PD \\
\hline 16 & 15 & Female & Femur & No & $\mathrm{LM}+\mathrm{BM}$ & 2 & PD \\
\hline 17 & 28 & Male & Humerus & No & LM & 2 & PD \\
\hline 18 & 20 & Male & Femur & No & $\mathrm{LM}+\mathrm{BM}$ & 2 & PD \\
\hline
\end{tabular}

*LM, lung metastasis; BM, bone metastasis; LR, local recurrence; PD, progression disease; PR, partial response; SD, stable disease

Table 3. Tumor Response to Continuous-Infusion Ifosfamide and Ddoxorubicin Combination at two Cycles

\begin{tabular}{lrc}
\hline Response & $\mathrm{n}$ & Percen \\
\hline Total assessable patient & 18 & 100 \\
CR & 0 & 0 \\
PR & 0 & 0 \\
SD & 4 & 22.3 \\
PD & 14 & 77.7 \\
Overall response rate (CR + PR) & 0 & \\
Tumor control (CR + PR + SD) & 4 & 22.3 \\
\hline
\end{tabular}

*CR, complete response; PR, partial response; PD, progression disease; $\mathrm{SD}$, stable disease

infusion ifosfamide and doxorubicin combination therapy (median, 2 cycles per patient; range: $2-5$ cycles) and a total of 42 cycles was administered. All of 18 patients were available for tumor response evaluation. After two cycles of treatment, 12 patients changed chemotherapy regimen due to disease progression, 2 patients refused to receive more chemotherapy due to personal or economic reasons, and 1 patient received five cycles of continuousinfusion ifosfamide and doxorubicin combination therapy.
We observed no CRs, no PRs, and four SDs (Table 2). The overall response rate and disease control rate were 0 and $22.3 \%$, respectively (Table 3 ). At the cut-off date, the median OS was 9 months (range: 3-29 months), and the median PFS was 2 months (range: 2-5 months).

We recorded no drug-related deaths. In general, chemotherapy-related adverse events were limited to Grade 1 or 2 . We noted the following Grade 3 and 4 toxic effects: leucopenia 7 (38.9\%), nausea and vomiting $3(16.7 \%)$ and alopecia $9(50 \%)$. Table 4 summarizes the main side effects and all Grade 3 and 4 adverse events.

\section{Discussion}

There is currently no standard salvage treatment as second-line chemotherapy for recurrent or refractory osteosarcoma. Several new agents such as topotecan (Seibel et al., 2007), irinotecan (Crews et al., 2004; Bagatell et al., 2014), imatinib mesylate (Bond et al., 2008), gefitinib (Brennan et al., 2014), trabectedin (Chuk et al., 2012), pirarubicin (Qi et al., 2012), ifosfamide (Choeyprasert et al., 2014; Li et al., 2014) and gemcitabine (Qi et al., 2012) have been investigated in pediatric and

Table 4. Hematologic and Non-hematologic Toxicities

\begin{tabular}{|c|c|c|c|c|c|c|c|c|}
\hline \multirow[t]{3}{*}{ Adverse event } & \multicolumn{6}{|c|}{ Grade } & & \\
\hline & \multicolumn{2}{|c|}{1 or 2} & \multicolumn{2}{|c|}{3} & \multicolumn{2}{|c|}{4} & \multicolumn{2}{|c|}{ Total } \\
\hline & $\mathrm{n}$ & $\%$ & $\mathrm{n}$ & $\%$ & $\mathrm{n}$ & $\%$ & $\mathrm{n}$ & $\%$ \\
\hline Anemia & 5 & 27.8 & 0 & 0 & 0 & 0 & 5 & 27.8 \\
\hline Leucopenia & 11 & 61.1 & 4 & 22.2 & 3 & 16.7 & 18 & 100 \\
\hline Thrombocytopenia & 3 & 16.7 & 0 & 0 & 0 & 0 & 3 & 16.7 \\
\hline Nausea and vomiting & 13 & 72.2 & 3 & 16.7 & 0 & 0 & 16 & 88.9 \\
\hline Fatigue & 5 & 27.8 & 0 & 0 & 0 & 0 & 5 & 27.8 \\
\hline Alopecia & 9 & 50 & 9 & 50 & 0 & 0 & 18 & 100 \\
\hline Impaired liver function & 2 & 11.1 & 0 & 0 & 0 & 0 & 2 & 11.1 \\
\hline Impaired kidney function & 0 & 0 & 0 & 0 & 0 & 0 & 0 & 0 \\
\hline Cardiotoxicity & 0 & 0 & 0 & 0 & 0 & 0 & 0 & 0 \\
\hline Hematuresis & 1 & 5.6 & 0 & 0 & 0 & 0 & 1 & 5.6 \\
\hline
\end{tabular}


adult patients, but the response rate is low and survival time is short. As a result, we undertook this retrospective study to investigate the activity of continuous-infusion ifosfamide and doxorubicin combination as secondline therapy for patients with relapsing and refractory osteosarcoma.

Of the 18 patients available for response evaluation in this retrospective study, we found that the overall response rate and the tumor control rate for relapsing or refractory osteosarcoma were 0 and $22.3 \%$, respectively. No patient achieved CR or PR, and four patients who achieved SD were all died from disease progression. At the cut-off date, the median OS was 9 months (rang: 3-29 months), and the median PFS was 2 months (rang: 2-5 months), which confirmed the poor prognosis of the disease.

Similar to other studies, the present study showed some grade 3-4 toxic events, such as leucopenia 7 (38.9\%), nausea and vomiting $3(16.7 \%)$ and alopecia $9(50 \%)$, but these toxic events were resolved by using supportive treatments such as G-CSF, interleukin-11, and 5-HT3 receptor blockers (as antiemetics).

To our knowledge, our trial is the first study to assess the efficacy and safety of continuous-infusion ifosfamide as second-line chemotherapy in recurrent or refractory osteosarcoma. However, we also acknowledge that the present study was limited by its retrospective nature, the small number of patients, and possible patient selection bias. What's more, there were no guidelines to follow for the staging and timing of imaging evaluations in our institution. Despite these limitations, we conducted this study to explore a completely different strategy in a rare sarcoma for which there were no other therapeutic options. And our study suggested that the continuous-infusion ifosfamide and doxorubicin combination therapy were well tolerated and moderate effective, which could be considered as salvage therapy for patients with recurrent or refractory osteosarcoma. However, because of short follow-up time, we could not observe the long-term results of this combination therapy, which should be confirmed in future studies.

\section{Acknowledgements}

Yao Yang and Huang Yujing were responsible for the study design, article drafting and data analysis. He Aina and Min Daliu were responsible for study concept, interpretation of data and critical appraisal. Shen Zan and Sun Yuanjue were responsible for revision of the article and critical appraisal.

\section{References}

Bacci G, Ferrari S, Lari S, et al (2002). Osteosarcoma of the limb. Amputation or limb salvage in patients treated by neoadjuvant chemotherapy. J Bone Joint Surg Br, 84, 88-92.

Bagatell R, Norris R, Ingle AM, et al (2014). Phase 1 trial of temsirolimus in combination with irinotecan and temozolomide in children, adolescents and young adults with relapsed or refractory solid tumors: a Children's Oncology Group Study. Pediatr Blood Cancer, 61, 833-9.

Bielack SS, Kempf-Bielack B, Delling G, et al (2002). Prognostic factors in high-grade osteosarcoma of the extremities or trunk: an analysis of 1,702 patients treated on neoadjuvant cooperative osteosarcoma study group protocols. J Clin Oncol, 20, 776-90.

Bond M, Bernstein ML, Pappo A, et al (2008). A phase II study of imatinib mesylate in children with refractory or relapsed solid tumors: a Children's Oncology Group study. Pediatr Blood Cancer, 50, 254-8.

Brennan RC, Furman W, Mao S, et al (2014). Phase I dose escalation and pharmacokinetic study of oral gefitinib and irinotecan in children with refractory solid tumors. Cancer Chemother Pharmacol, 74, 1191-8.

Cartei G, Clocchiatti L, Sacco C, et al (2003). Dose finding of ifosfamide administered with a chronic two-week continuous infusion. Oncology, 65, 31-6.

Choeyprasert W, Pakakasama S, Sirachainan N, et al (2014). Comparative outcome of Thai pediatric osteosarcoma treated with two protocols: the role of high-dose methotrexate (HDMTX) in a single institute experience. Asian Pac J Cancer Prev, 15, 9823-9.

Chuk MK, Aikin A, Whitcomb T, et al (2012). A phase I trial and pharmacokinetic study of a 24-hour infusion of trabectedin (Yondelis (R), ET-743) in children and adolescents with relapsed or refractory solid tumors. Pediatr Blood Cancer, 59, 865-9.

Crews KR, Stewart CF, Liu T, et al (2004). Effect of fractionated ifosfamide on the pharmacokinetics of irinotecan in pediatric patients with osteosarcoma. J Pediatr Hematol Oncol, 26, 764-7.

De Pas T, Rosati G, Spitaleri G, et al (2011). Optimizing clinical care in patients with advanced soft tissue sarcoma: a phase II study of a new schedule of high-dose continuous infusion ifosfamide and doxorubicin combination. Chemotherapy, 57, 217-24.

Ferrari S, Briccoli A, Mercuri M, et al (2003). Postrelapse survival in osteosarcoma of the extremities: prognostic factors for long-term survival. J Clin Oncol, 21, 710-5.

Harris MB, Cantor AB, Goorin AM, et al (1995). Treatment of osteosarcoma with ifosfamide: comparison of response in pediatric patients with recurrent disease versus patients previously untreated: a Pediatric Oncology Group study. Med Pediatr Oncol, 24, 87-92.

Hawkins DSand Arndt CA (2003). Pattern of disease recurrence and prognostic factors in patients with osteosarcoma treated with contemporary chemotherapy. Cancer, 98, 2447-56.

Kobys VL, Konovalenko VF, Repinsmall a CNV, et al (2013). Treatment of large osteosarcoma in children: new approach. Exp Oncol, 35, 105-8.

Kudawara I, Aoki Y, Ueda T, et al (2013). Neoadjuvant and adjuvant chemotherapy with high-dose ifosfamide, doxorubicin, cisplatin and high-dose methotrexate in nonmetastatic osteosarcoma of the extremities: a phase II trial in Japan. J Chemother, 25, 41-8.

Li YY, Jiang XM, Dong YG, Xu G, Ma YB (2014). Ifosfamidecontaining regimens for treating patients with osteosarcomas. Asian Pac J Cancer Prev, 15, 9763-6.

Meyers PA, Schwartz CL, Krailo M, et al (2005). Osteosarcoma: a randomized, prospective trial of the addition of ifosfamide and/or muramyl tripeptide to cisplatin, doxorubicin, and high-dose methotrexate. J Clin Oncol, 23, 2004-11.

Palumbo R, Palmeri S, Antimi M, et al (1997). Phase II study of continuous-infusion high-dose ifosfamide in advanced and/ or metastatic pretreated soft tissue sarcomas. Ann Oncol, 8, 1159-62.

Qi WX, He AN, Tang LN, et al (2012). Efficacy and safety of gemcitabine-docetaxel combination therapy for recurrent or refractory high-grade osteosarcoma in China: a retrospective study of 18 patients. Jpn J Clin Oncol, 42, 427-31. 
Qi WX, He AN, Tang LN, et al (2012). Evaluation of pirarubicincisplatin chemotherapy in the treatment for refractory and recurrent high-grade osteosarcoma: experience of a single institute. Med Oncol, 29, 2229-33.

Saeter G, Hoie J, Stenwig AE, et al (1995). Systemic relapse of patients with osteogenic sarcoma. Prognostic factors for long term survival. Cancer, 75, 1084-93.

Seibel NL, Krailo M, Chen Z, et al (2007). Upfront window trial of topotecan in previously untreated children and adolescents with poor prognosis metastatic osteosarcoma: children's Cancer Group (CCG) 7943. Cancer, 109, 1646-53.

Singer JM, Hartley JM, Brennan C, et al (1998). The pharmacokinetics and metabolism of ifosfamide during bolus and infusional administration: a randomized cross-over study. Br J Cancer, 77, 978-84.

Sluga M, Windhager R, Lang S, et al (1999). Local and systemic control after ablative and limb sparing surgery in patients with osteosarcoma. Clin Orthop Relat Res, 120-7.

Ta HT, Dass CR, Choong PF, et al (2009). Osteosarcoma treatment: state of the art. Cancer Metastasis Rev, 28, 247-63.

Therasse P, Arbuck SG, Eisenhauer EA, et al (2000). New guidelines to evaluate the response to treatment in solid tumors. european organization for research and treatment of cancer, National Cancer Institute of the United States, National Cancer Institute of Canada. J Natl Cancer Inst, 92, 205-16.

Wilkins RM, Cullen JW, Odom L, et al (2003). Superior survival in treatment of primary nonmetastatic pediatric osteosarcoma of the extremity. Ann Surg Oncol, 10, 498-507. 